ANNALES

POLONICI MATHEMATICI

$88.1(2006)$

\title{
Non-isotropic Hausdorff capacity of exceptional sets for pluri-Green potentials in the unit ball of $\mathbb{C}^{n}$
}

\author{
by Kuzman Adzievski (Orangeburg, SC)
}

\begin{abstract}
We study questions related to exceptional sets of pluri-Green potentials $V_{\mu}$ in the unit ball $B$ of $\mathbb{C}^{n}$ in terms of non-isotropic Hausdorff capacity. For suitable measures $\mu$ on the ball $B$, the pluri-Green potentials $V_{\mu}$ are defined by

$$
V_{\mu}(z)=\int_{B} \log \frac{1}{\left|\phi_{z}(w)\right|} d \mu(w),
$$

where for a fixed $z \in B, \phi_{z}$ denotes the holomorphic automorphism of $B$ satisfying $\phi_{z}(0)=z, \phi_{z}(z)=0$ and $\left(\phi_{z} \circ \phi_{z}\right)(w)=w$ for every $w \in B$. If $d \mu(w)=f(w) d \lambda(w)$, where $f$ is a non-negative measurable function of $B$, and $\lambda$ is the measure on $B$, invariant under all holomorphic automorphisms of $B$, then $V_{\mu}$ is denoted by $V_{f}$. The main result of this paper is as follows: Let $f$ be a non-negative measurable function on $B$ satisfying

$$
\int_{B}\left(1-|z|^{2}\right) f^{p}(z) d \lambda(z)<\infty
$$
\end{abstract}

for some $p$ with $1<p<n /(n-1)$ and some $\alpha$ with $0<\alpha<n+p-n p$. Then for each $\tau$ with $1 \leq \tau \leq n / \alpha$, there exists a set $E_{\tau} \subseteq S$ with $H_{\alpha \tau}\left(E_{\tau}\right)=0$ such that

$$
\lim _{\substack{z \rightarrow \zeta \\ z \in \mathcal{T}_{\tau, c}(\zeta)}} V_{f}(z)=0
$$

for all points $\zeta \in S \backslash E_{\tau}$. In the above, for $\alpha>0, H_{\alpha}$ denotes the non-isotropic Hausdorff capacity on $S$, and for $\zeta \in S=\partial B, \tau \geq 1$, and $c>0, \mathcal{T}_{\tau, c}(\zeta)$ are the regions defined by

$$
\mathcal{T}_{\tau, c}(\zeta)=\left\{z \in B:|1-\langle z, \zeta\rangle|^{\tau}<c\left(1-|z|^{2}\right)\right\} .
$$

1. Introduction. Exceptional sets of Blaschke products, holomorphic functions, or Green potentials in the unit disc of the complex plane are usually described in terms of Hausdorff capacity (measure). For example, Samuelsson [15] and Cargo [8] studied exceptional sets of Blaschke products

2000 Mathematics Subject Classification: 31B25, 31C15, 32F99.

Key words and phrases: non-isotropic Hausdorff capacity, pluri-Green potentials, Blaschke product, tangential limits. 
in terms of Hausdorff capacity. In [7], Berman and Cohn prove several results concerning the existence of certain non-tangential and tangential limits of Blaschke products and their subproducts in terms of Hausdorff capacity. On the other hand, in higher dimension, the non-isotropic Hausdorff capacity is much more appropriate for the description of exceptional sets of holomorphic functions, or invariant Green potentials.

Many authors have investigated the non-isotropic Hausdorff capacity as a tool for the description of exceptional sets of some function classes. For example, in [17] and [18], Stoll investigated some questions related to the nonisotropic Hausdorff capacity of exceptional sets of invariant Green potentials in the unit ball $B$ of $\mathbb{C}^{n}$. In [4], Ahern and Cohn, using the non-isotropic Hausdorff capacity, studied exceptional sets for Hardy-Sobolev functions in the unit ball in $\mathbb{C}^{n}$. Also, in [5], using $L^{p}$-estimates for maximal functions of invariant Poisson integrals Ahern and Cohn derived that the exceptional sets of the Poisson integrals of potentials are sets of zero Hausdorff measure. Ahern and Cascante [3] investigated exceptional sets for Poisson integrals of potentials on the unit sphere in $\mathbb{C}^{n}$. Some results for exceptional sets with zero non-isotropic Hausdorff measure for holomorphic Sobolev functions in the unit ball of $\mathbb{C}^{n}$ are given in [2] by Ahern. In [11], Cohn gave a characterization of exceptional sets for a class of holomorphic functions in terms of non-isotropic Hausdorff capacities. Ahern and Nagel [6] studied, among many other things, the existence of tangential limits of Poisson integrals of $L^{p}$-potentials and functions in Besov spaces within tangential approach regions in terms of appropriate Hausdorff measure. Cascante and Ortega [9] characterized some closed tangential exceptional sets for Hardy-Sobolev spaces in the unit ball of $\mathbb{C}^{n}$ in terms of non-isotropic Hausdorff measure. The main goal of Jamming and Roginskaya [12] and Roginskaya [13] is to describe the singular part of a measure on the unit sphere $S$ in $\mathbb{C}^{n}$ in terms of behavior of its harmonic and $\mathcal{M}$-harmonic extension near the boundary. Their description involves comparisons of the Hausdorff measure and the non-isotropic Hausdorff measure, associated to the isotropic Euclidean and non-isotropic (Korányi) distance on $S$, respectively.

Homogeneous spaces, in connection with Hausdorff measure and boundary behavior of some function classes have been studied by many authors. For example, Sjödin [16] investigates analogues of classical Riesz capacity and Hausdorff measure in a homogeneous space. Since the unit sphere $S$ in $\mathbb{C}^{n}$, with the Korányi distance, is a space of homogeneous type, the results of his paper can be applied for this metric space. In [10], Cifuentes, Dorronsoro and Sueiro proved strong $L^{p}$ estimates for weighted maximal functions of Poisson integrals on spaces of homogeneous type with respect to Hausdorff content. Sueiro [20] studies questions related to Fatou's theorem and "fractional Cauchy kernel" for the unit ball $B$ in $\mathbb{C}^{n}$. By studying 
weighted maximal functions in metric spaces of homogeneous type he obtained estimates on fractional Cauchy kernel and a strong-type maximal estimate. He then used these estimates to obtain a result on the Hausdorff measure, defined by the Korányi balls, of the exceptional set of boundary limits of the fractional Cauchy integrals.

Let $\mathcal{B}$ be the class of all Blaschke products on the open unit disc $U$ of the complex plane $\mathbb{C}$, i.e., all functions of the form

$$
\beta(z)=z^{m} \prod_{n=1}^{\infty} \frac{\bar{a}_{n}}{a_{n}} \cdot \frac{a_{n}-z}{1-\bar{a}_{n} z},
$$

where $m$ is a nonnegative integer and $\left\{a_{n}\right\}$ is a sequence of non-zero complex numbers in $U$ which satisfies the condition

$$
\sum_{n=1}^{\infty}\left(1-\left|a_{n}\right|\right)<\infty .
$$

The class of all non-decreasing, continuous functions $h$ on $[0, \infty)$ such that $h(t)>0$ if $t>0$ and $h(t) / t$ is non-increasing on $(0, \infty)$ will be denoted by $\mathcal{H}$. Examples of such functions are $h(t)=t^{\alpha}, 0 \leq \alpha \leq 1$. The subclass of $\mathcal{H}$, consisting of the functions $h \in \mathcal{H}$ satisfying the additional condition $h(0)=0$, is denoted by $\mathcal{H}_{0}$.

If $h \in \mathcal{H}_{0}$, then $h$ is a determining function for a Hausdorff capacity (measure) $H_{h}$ defined on the compact subsets $K$ of the boundary $\partial U$ of $U$ by

$$
H_{h}(K)=\inf \left\{\sum_{j=1}^{\infty} h\left(r_{j}\right)\right\},
$$

where the infimum is taken over all countable covers $\left\{D\left(\zeta_{j}, r_{j}\right): j=1,2, \ldots\right\}$ of $K$ by discs $D\left(\zeta_{j}, r_{j}\right)$ centered at $\zeta_{j} \in \partial U$ and of radius $r_{j}$. For an arbitrary subset $A$ of $\partial U$, the Hausdorff capacity $H_{h}$ is defined by

$$
H_{h}(A)=\sup \left\{H_{h}(K): K \text { a compact subset of } A\right\} .
$$

Following Samuelsson's paper [15], for $\beta \in \mathcal{B}, \zeta \in \partial U$, and $t>0$ let

$$
\sigma(\beta, \zeta, t)=\sum_{\left|a_{k}-\zeta\right| \leq t}\left(1-\left|a_{k}\right|\right) .
$$

Further, for $h \in \mathcal{H}, \beta \in \mathcal{B}$ we need the sets

$$
\begin{aligned}
& \mathcal{L}(\beta, h)=\left\{\zeta \in \partial U: \liminf _{r \rightarrow 1-} \frac{1-r}{h(1-r)} \log \frac{1}{|\beta(r \zeta)|}=+\infty\right\}, \\
& \underline{\Sigma}(\beta, h)=\left\{\zeta \in \partial U: \liminf _{t \rightarrow 0+} \frac{\sigma(\beta, \zeta, t)}{h(t)}=+\infty\right\},
\end{aligned}
$$




$$
\begin{aligned}
\bar{\Sigma}(\beta, h) & =\left\{\zeta \in \partial U: \limsup _{t \rightarrow 0+} \frac{\sigma(\beta, \zeta, t)}{h(t)}=+\infty\right\}, \\
\mathcal{L}_{0}(\beta, h) & =\left\{\zeta \in \partial U: \liminf _{r \rightarrow 1-} \frac{1-r}{h(1-r)} \log \frac{1}{\beta(r \zeta)}>0\right\} .
\end{aligned}
$$

In like manner, for $0<\alpha<1$ let

$$
\mathcal{L}_{\alpha}(\beta, h)=\left\{\zeta \in \partial U: \liminf _{\substack{z \rightarrow \zeta \\ z \in S_{\alpha}(\zeta)}} \frac{|z-\zeta|}{h(|z-\zeta|)} \log \frac{1}{|\beta(z)|}=+\infty\right\}
$$

for all Stolz domains $S_{\alpha}(\zeta)$ defined by

$$
S_{\alpha}(\zeta)=\left\{z \in U:|z-\zeta| \leq \sqrt{\alpha-\alpha^{2}},|\arg (1-\bar{\zeta} z)| \leq \arcsin \alpha\right\} .
$$

In [15], Samuelsson proved the following results:

THEOREm A. If $\beta$ is a Blaschke product, then the set $\mathcal{L}_{0}(\beta, 1)$ is empty. If $h \in \mathcal{H}_{0}$, then

$$
H_{h}\left(\mathcal{L}_{0}(\beta, h)\right)=0 .
$$

Theorem B. Let $h \in \mathcal{H}$ and let $\beta \in \mathcal{B}$. Then

$$
\underline{\Sigma}(\beta, h) \subseteq \mathcal{L}_{\alpha}(\beta, h) \subseteq \mathcal{L}(\beta, h) \subseteq \bar{\Sigma}(\beta, h) .
$$

This paper was motivated by the work of Stoll [18], concerning the nonisotropic Hausdorff capacity of invariant Green potentials $G_{\mu}$ on the unit ball $B$ of $\mathbb{C}^{n}$. For $\zeta \in S=\partial B, \tau \geq 1$, and $c>0$, let

$$
\mathcal{T}_{\tau, c}(\zeta)=\left\{z \in B:|1-\langle z, \zeta\rangle|^{\tau}<c\left(1-|z|^{2}\right)\right\} .
$$

The regions $\mathcal{T}_{\tau, c}(\zeta)$ have tangential contact with the boundary $S$ of the ball $B$ in all directions at the boundary point $\zeta \in S$.

When $\tau=1$ and $2 c=\alpha>1$ the regions $\mathcal{T}_{\tau, c}(\zeta)$ reduce to the admissible domains of Korányi

$$
D_{\alpha}(\zeta)=\left\{z \in B:|1-\langle z, \zeta\rangle|<\frac{\alpha}{2}\left(1-|z|^{2}\right)\right\} .
$$

A function $V$ on $B$ is said to have a $\mathcal{T}_{\tau}$-limit $L$ at $\zeta \in S$ if

$$
\lim _{\substack{z \rightarrow \zeta \\ z \in \mathcal{T}_{\tau, c}(\zeta)}} V(z)=L
$$

for every $c>1(c>1 / 2$ when $\tau=1)$.

The exceptional set for a function $f$ defined on the unit ball $B$ is the set of all points $\zeta \in S$ such that $f$ fails to have a $\mathcal{T}_{\tau}(\zeta)$-limit at $\zeta$.

In [18], Stoll proved the following result concerning tangential limits and exceptional sets: 
TheOREM C. Let $f$ be a non-negative measurable function on $B$ satisfying

$$
\int_{B}\left(1-|z|^{2}\right)^{\beta} f^{p}(z) d \lambda(z)<\infty
$$

for some $p>n$ and some $\beta$ with $0<\beta<n$. Then for each $\tau$ with $1 \leq$ $\tau \leq n / \beta$, there exists a set $E_{\tau} \subseteq S$ with $H_{\beta \tau}\left(E_{\tau}\right)=0$ such that $G_{f}$ has $\mathcal{T}_{\tau}(\zeta)$-limit 0 at all $\zeta \in S \backslash E_{\tau}$.

In the above, for $0<\alpha<n, H_{\alpha}$ denotes the non-isotropic Hausdorff capacity on $S, G_{f}$ denotes the Green potential of $f$, and $\lambda$ denotes a measure on $B$ invariant under the group of all holomorphic automorphisms of the ball $B$.

The methods used to prove Theorem $\mathrm{C}$ allowed Stoll to prove the following result:

Theorem D. Let $\left\{a_{j}\right\}$ be a sequence in B satisfying

$$
\sum_{j=1}^{\infty}\left(1-\left|a_{j}\right|^{2}\right)^{\beta}<\infty
$$

for some $\beta$ with $0<\beta<n$, and let $\mu$ be the measure given by $\mu=\sum \delta_{a_{j}}$, where $\delta_{a_{j}}$ is the unit point-mass measure at $a_{j}$. Then for each $\tau$ with $1 \leq$ $\tau \leq n / \beta$, there exists a set $E_{\tau} \subseteq S$ with $H_{\beta \tau}\left(E_{\tau}\right)=0$ such that $G_{\mu}$ has $\mathcal{T}_{\tau}$-limit 0 at all $\zeta \in S \backslash E_{\tau}$.

In the above theorem, $G_{\mu}$ denotes the invariant Green potential of the measure $\mu$ on the unit ball $B$.

The purpose of our paper is to investigate analogues of Stoll's results (Theorems $\mathrm{C}$ and D) for pluri-Green potentials in the unit ball of $\mathbb{C}^{n}$. We also extend Samuelsson's results (Theorems A and B) by considering nonisotropic Hausdorff capacity of pluri-Green potentials.

The paper is organized as follows. In Section 2, we introduce the necessary notation and definitions. In Section 3, we give some preliminary results that will be used for the proofs of the main results which are given in Section 4 .

This paper is part of the author's dissertation written under the direction of Professor Manfred Stoll at the University of South Carolina.

2. Notation. Throughout this paper we use the notation and terminology of Rudin's book [14] and most of the general results used in our paper can be found in that book. For $n \geq 1, \mathbb{C}^{n}$ denotes the $n$-dimensional complex space, with the usual inner product $\langle z, w\rangle$ and norm $|z|=\sqrt{\langle z, z\rangle}$. $B$ will denote the unit ball in $\mathbb{C}^{n}$ with $d \nu$ the Lebesgue measure on $B$ normalized 
so that $\nu(B)=1$, and $S=\partial B$ will be the boundary of $B$ with $d \sigma$ the surface area measure on $S$, again normalized so that $\sigma(S)=1$.

For $\delta>0$ and $\zeta \in S$ let

$$
Q(\zeta, \delta)=\{\eta \in S:|1-\langle\eta, \zeta\rangle|<\delta\}
$$

REMARK. The set $Q(\zeta, \delta)$ is called a Korányi ball.

The following fact, whose proof can be found in [14, p. 66], will be used:

Proposition K. The function

$$
d(w, z)=\sqrt{|1-\langle w, z\rangle|}, \quad w, z \in \bar{B},
$$

satisfies the triangle inequality

$$
d(w, z) \leq d(w, x)+d(x, z) \quad \text { for all } w, x, z \in \bar{B} .
$$

Restricted to the sphere $S$, the function $d$ is a metric, and the sets $Q(\zeta, \delta)$ are the corresponding open balls.

Let $h$ be a non-decreasing function on $[0, \infty)$ vanishing at 0 and satisfying the condition

$$
h(2 x) \leq \operatorname{ch}(x)
$$

for some positive constant $c$. Such a function $h$ will be referred to as allowed.

REMARK. It is easy to check that each $h \in \mathcal{H}_{0}$ is an allowed function.

For an allowed function $h$, the non-isotropic Hausdorff capacity $H_{h}$ of a compact subset $K$ of $S$ is defined by

$$
H_{h}(K)=\inf \left\{\sum_{j=1}^{\infty} h\left(r_{j}\right)\right\},
$$

where the infimum is taken over all countable covers $\left\{Q\left(\zeta_{j}, r_{j}\right): j \in \mathbb{N}\right\}$ of $K$ by Korányi balls $Q\left(\zeta_{j}, r_{j}\right)$ centered at $\zeta_{j} \in S$ and of radius $r_{j}$. For an arbitrary subset $A$ of $S$, the non-isotropic Hausdorff capacity $H_{h}$ is defined by

$$
H_{h}(A)=\sup \left\{H_{h}(K): K \text { a compact subset of } A\right\} \text {. }
$$

For basic definitions, background and more information on the nonisotropic Hausdorff capacity we refer to [12], [13] and [16].

For each $z \in B, \phi_{z}$ denotes the holomorphic automorphism of $B$ satisfy$\operatorname{ing} \phi_{z}(0)=z, \phi_{z}(z)=0$ and $\phi_{z} \circ \phi_{z}(w)=w$ for every $w \in B$. The following identity will be useful throughout:

$$
1-\left|\phi_{a}(z)\right|^{2}=\frac{\left(1-|z|^{2}\right)\left(1-|a|^{2}\right)}{|1-\langle z, a\rangle|^{2}}
$$

for all $a \in B$ and $z \in \bar{B}$. 
$\mathcal{M}$ will denote the Möbius group (of all holomorphic automorphisms) of the ball $B$. By Cartan's theorem it follows that $\psi \in \mathcal{M}$ if and only if $\psi=u \circ \phi_{a}$ for a unique unitary transformation $u$ on $\mathbb{C}^{n}$, where $a=\psi^{-1}(0)$.

For $r>0$, let

$$
B_{r}=\left\{z \in \mathbb{C}^{n}:|z|<r\right\},
$$

and for $a \in B, a \neq 0$, let

$$
E(a)=\phi_{a}\left(B_{1 / 2}\right) .
$$

Since $\phi_{a}$ is an involution, $z \in E(a)$ if and only if $\left|\phi_{a}(z)\right|<1 / 2$.

We denote by $\lambda$ the measure on $B$ defined by

$$
d \lambda(z)=\frac{d \nu(z)}{\left(1-|z|^{2}\right)^{n+1}} .
$$

This measure is $\mathcal{M}$-invariant, i.e.

$$
\int_{B} f(z) d \lambda(z)=\int_{B}(f \circ \psi)(z) d \lambda(z)
$$

for each $f \in L^{1}(d \lambda)$ and all $\psi \in \mathcal{M}$.

The Laplace-Beltrami operator (invariant Laplacian) $\widetilde{\Delta}$ on $B$ is given by

$$
\widetilde{\Delta} f(z)=\frac{1}{n+1} \Delta\left(f \circ \phi_{z}\right)(0)=\frac{4\left(1-|z|^{2}\right)}{n+1} \sum_{i, j=1}^{n}\left(\delta_{i, j}-z_{i} \bar{z}_{j}\right) \frac{\partial^{2} f}{\partial z_{j} \partial \bar{z}_{i}},
$$

where

$$
\delta_{i, j}= \begin{cases}1 & \text { for } i=j, \\ 0 & \text { for } i \neq j,\end{cases}
$$

and $\Delta$ is the usual Laplacian in $\mathbb{R}^{2 n}$.

The Green function for the Laplace-Beltrami operator $\widetilde{\Delta}$ is given by

$$
G(z, w)=g\left(\phi_{z}(w)\right)
$$

where

$$
g(z)=\frac{n+1}{2 n} \int_{|z|}^{1}\left(1-t^{2}\right)^{n-1} t^{-2 n+1} d t .
$$

Remark. For $n=1, g(z)=-\log (|z|)$, and the Green function for the Laplace-Beltrami operator $\widetilde{\Delta}$ coincides with the usual Green function $G$ on the unit $\operatorname{disc} U$.

If $\mu$ is a non-negative regular Borel measure on $B$, then the function

$$
G_{\mu}(z)=\int_{B} G(z, w) d \mu(w)
$$


is called the invariant Green potential of $\mu$ on $B$, provided

$$
\int_{B} G\left(z_{0}, w\right) d \mu(w)<\infty
$$

for some $z_{0} \in B$. The above condition is satisfied if and only if

$$
\int_{B}\left(1-|w|^{2}\right)^{n} d \mu(w)<\infty .
$$

For each fixed $w \in B$, the function

$$
z \mapsto \log \frac{1}{\left|\phi_{z}(w)\right|}
$$

is called the pluri-Green function of $B$ with pole at $w$. When $n=1$,

$$
\log \frac{1}{\left|\phi_{a}(z)\right|}=\log \left|\frac{1-z \bar{a}}{z-a}\right|,
$$

which is the usual Green function on $U$.

If $\mu$ is a non-negative regular Borel measure on $B$ then the function

$$
V_{\mu}(z)=\int_{B} \log \frac{1}{\left|\phi_{z}(w)\right|} d \mu(w)
$$

is called the invariant pluri-Green potential of $\mu$ on $B$, provided

$$
\int_{B} \log \frac{1}{\left|\phi_{z_{0}}(w)\right|} d \mu(w)<\infty,
$$

for some $z_{0} \in B$. In [1] it is shown that this is equivalent to

$$
\int_{B}\left(1-|w|^{2}\right) d \mu(w)<\infty .
$$

For $\zeta \in S$ and $t>0$ let

$$
B_{t}(\zeta)=\{z \in B:|1-\langle z, \zeta\rangle| \leq t\},
$$

and for a non-negative regular Borel measure $\mu$ satisfying the growth condition (2.2) let

$$
r(\mu, \zeta, t)=\int_{B_{t}(\zeta)}\left(1-|w|^{2}\right) d \mu(w) .
$$

For a regular Borel measure $\mu$ on $B$ satisfying the growth condition (2.2), and for $h \in \mathcal{H}$ and $0<\alpha<1$ we introduce the sets

$$
\begin{aligned}
\mathcal{L}(\mu, h) & =\left\{\zeta \in S: \liminf _{r \rightarrow 1-} \frac{1-r}{h(1-r)} V_{\mu}(r \zeta)=+\infty\right\}, \\
\mathcal{L}_{\alpha}(\mu, h) & =\left\{\zeta \in S: \liminf _{\substack{z \rightarrow \zeta \\
z \in D_{\alpha}(\zeta)}} \frac{|1-\langle z, \zeta\rangle|}{h(|1-\langle z, \zeta\rangle|)} V_{\mu}(z)=+\infty\right\},
\end{aligned}
$$




$$
\begin{aligned}
\underline{\mathcal{R}}(\mu, h) & =\left\{\zeta \in S: \liminf _{t \rightarrow 0+} \frac{r(\mu, \zeta, t)}{h(t)}=+\infty\right\}, \\
\overline{\mathcal{R}}(\mu, h) & =\left\{\zeta \in S: \limsup _{t \rightarrow 0^{+}} \frac{r(\mu, \zeta, t)}{h(t)}=+\infty\right\}, \\
\mathcal{L}_{0}(\mu, h) & =\left\{\zeta \in S: \liminf _{r \rightarrow 1-} \frac{1-r}{h(1-r)} V_{\mu}(r \zeta)>0\right\} .
\end{aligned}
$$

A final remark on notation: throughout this paper we will use the same letter $C$ or $C(a, b)$ to denote various absolute positive constants or positive constants which depend only on the indicated variables, but not necessarily the same on any two occurrences.

3. Preliminary results. In this section several preliminary results are given that will be used for the proof of the main theorems.

For $z \in B, c$ a real number, and $\alpha>n$ consider

$$
J_{c, \alpha}(z)=\int_{B} \frac{\left(1-|w|^{2}\right)^{\alpha}}{|1-\langle z, w\rangle|^{\alpha+c}} d \lambda(w) .
$$

The following asymptotic estimate will play an important role in the proof of our main results; its proof can be found in [14, pp. 17-18].

Proposition 3.1.

$$
J_{c, \alpha}(z) \approx \begin{cases}\left(1-|z|^{2}\right)^{-c}, & c>0 \\ \log \frac{1}{1-|z|^{2}}, & c=0, \\ 1, & c<0 .\end{cases}
$$

The notation $a(z) \approx b(z)$ means that the ratio $a(z) / b(z)$ has a finite limit as $|z| \rightarrow 0$.

The next, "Frostman type" theorem (Theorem 1 in [11], proved for $h(t)$ $\left.=t^{m}\right)$ is the key to the proof of our main results. The extension to arbitrary allowed $h$ (see [2]) poses no difficulty.

THEOREM 3.2. Let $h$ be an allowed function. For a compact subset $K$ of $S, H_{h}(K)>0$ if and only if $K$ contains the support of a positive measure $\nu \not \equiv 0$ satisfying

$$
\nu(Q(\zeta, \delta)) \leq C h(\delta)
$$

for all $\delta>0$ and $\zeta \in S$ and an absolute constant $C$.

The following covering lemma (see [14, Lemma 5.2.3]) will also be needed:

Lemma 3.3. Suppose that $E$ is the union of a finite collection $\left\{Q\left(\zeta_{i}, \delta_{i}\right)\right\}$ of Korányi balls. Then there exists a finite disjoint subcollection 
$\left\{Q\left(\zeta_{i_{k}}, \delta_{i_{k}}\right)\right\}_{k=1}^{m}$ such that

$$
E \subseteq \bigcup_{k=1}^{m} Q\left(\zeta_{i_{k}}, 9 \delta_{i_{k}}\right) \quad \text { and } \quad \sigma(E) \leq C_{n} \sum_{k=1}^{m} \sigma\left(Q\left(\zeta_{i_{k}}, \delta_{i_{k}}\right)\right),
$$

where $C_{n}$ is a constant depending only on $n$.

On several occasions in the paper we shall use the following elementary inequality:

$$
\log x \leq x-1 \quad \text { for } x>0 .
$$

Now, we proceed to several other lemmas, analogous to those in [15].

LEMma 3.4. Let $t$ be a fixed number such that $0<t<1 / 3$ and let $I_{t}=[1-3 t, 1-2 t]$. For $\zeta \in S$ and $z \in B$ let

$$
G_{\zeta, t}(z)=\int_{I_{t}} \log \frac{1}{\left|\phi_{z}(r \zeta)\right|} d r .
$$

Then there exist positive constants $C_{1}$ and $C_{2}$, independent of $\zeta, z$ and $t$, such that

$$
G_{\zeta, t}(z) \leq C_{1}\left(1-|z|^{2}\right)
$$

and

$$
G_{\zeta, t}(z) \leq C_{2} t^{2} \frac{1-|z|}{|1-\langle z, \zeta\rangle|^{2}}
$$

for all $z \in B$.

Proof. First, suppose that $\zeta=e_{1}=(1, \ldots, 0)$. For $z \in B$, by the identity (2.1) we have

$$
\begin{aligned}
G_{e_{1}, t}(z) & =\frac{1}{2} \int_{I_{t}} \log \left\{1+\frac{\left(1-r^{2}\right)\left(1-|z|^{2}\right)}{\left|1-\left\langle z, r e_{1}\right\rangle\right|^{2}-\left(1-r^{2}\right)\left(1-|z|^{2}\right)}\right\} d r \\
& \leq \frac{1}{2} \int_{I_{t}} \log \left\{1+\frac{\left(1-r^{2}\right)\left(1-|z|^{2}\right)}{|| z|-r|^{2}}\right\} d r .
\end{aligned}
$$

CASE 1. If $\min \left\{|| z|-r|: r \in I_{t}\right\} \geq t$, then by (3.2) we have

$$
\frac{1}{2} \int_{I_{t}} \log \left\{1+\frac{12 t(1-|z|)}{t^{2}}\right\} d r \leq 6 \frac{1-|z|}{t} \int_{I_{t}} d r \leq 6(1-|z|),
$$

which proves (3.3) for $\zeta=e_{1}$ in this case.

CASE 2. If $\min \left\{|| z|-r|: r \in I_{t}\right\} \leq t$, then $1-|z|=1-r-(|z|-r)>$ $1-r-t \geq t$. Therefore in this case we have 


$$
\begin{aligned}
G_{e_{1}, t}(z) & \leq \frac{1}{2} \int_{I_{t}} \log \left\{1+\frac{6 t\left(1-|z|^{2}\right)}{|| z|-r|^{2}}\right\} d r \leq \frac{1}{2} \int_{I_{t}} \log \left\{1+\left(\frac{4(1-|z|)}{|| z|-r|}\right)^{2}\right\} d r \\
& \leq \int_{|z|}^{\infty} \log \left\{1+\left(\frac{4(1-|z|)}{|| z|-r|}\right)^{2}\right\} d r \\
& \leq 4(1-|z|) \int_{0}^{\infty} \frac{1}{x^{2}} \log \left(1+x^{2}\right) d x \leq C\left(1-|z|^{2}\right)
\end{aligned}
$$

which proves (3.3) for $\zeta=e_{1}$.

To prove (3.4) for $\zeta=e_{1}$, first notice that for $z=\left(z_{1}, \ldots, z_{n}\right) \in B$ we have

$$
\left|1-\left\langle z, r e_{1}\right\rangle\right|^{2}-\left(1-r^{2}\right)\left(1-|z|^{2}\right) \geq\left|z_{1}-r\right|^{2} \text {. }
$$

The last inequality is easily verified. Indeed, using the obvious inequality $|z| \geq\left|z_{1}\right|$ we have

$$
\begin{aligned}
\left|1-\left\langle z, r e_{1}\right\rangle\right|^{2}-\left(1-r^{2}\right)\left(1-|z|^{2}\right) & =\left|1-r z_{1}\right|^{2}-\left(1-r^{2}\right)\left(1-|z|^{2}\right) \\
& \geq\left|1-r z_{1}\right|^{2}-\left(1-r^{2}\right)\left(1-\left|z_{1}\right|^{2}\right) \\
& =\left|r-z_{1}\right|^{2}
\end{aligned}
$$

Now let $z=\left(z_{1}, \ldots, z_{n}\right) \in B$ be such that $\left|1-z_{1}\right|>4 t$. Then for $r \in I_{t}$,

$$
\begin{aligned}
\left|z_{1}-r\right| & \geq\left|1-z_{1}\right|-(1-r) \geq\left|1-z_{1}\right|-3 t \\
& >\left|1-z_{1}\right|-\frac{3}{4}\left|1-z_{1}\right|=\frac{1}{4}\left|1-z_{1}\right| .
\end{aligned}
$$

Therefore from (3.5) and (3.2) it follows that

$$
\begin{aligned}
G_{e_{1}, t}(z) & =\frac{1}{2} \int_{I_{t}} \log \left\{1+\frac{\left(1-r^{2}\right)\left(1-|z|^{2}\right)}{\left|1-\left\langle z, r e_{1}\right\rangle\right|^{2}-\left(1-r^{2}\right)\left(1-|z|^{2}\right)}\right\} d r \\
& \leq \frac{1}{2} \int_{I_{t}} \log \left\{1+\frac{12 t(1-|z|)}{\left|z_{1}-r\right|^{2}}\right\} d r \leq \frac{1}{2} \int_{I_{t}} \frac{(12 t)(1-|z|)}{\left|z_{1}-r\right|^{2}} d r \\
& \leq \frac{1}{2} \frac{(12 t)(16 t)(1-|z|)}{\left|1-z_{1}\right|^{2}}=96 t^{2} \frac{1-|z|}{\left|1-\left\langle z, e_{1}\right\rangle\right|^{2}} .
\end{aligned}
$$

If $\left|1-z_{1}\right|<4 t$, then by (3.3) for $\zeta=e_{1}$, we have

$$
\begin{aligned}
G_{e_{1}, t}(z) & \leq C_{1}\left(1-|z|^{2}\right)=C_{1}\left(1-|z|^{2}\right) \frac{\left|1-z_{1}\right|^{2}}{1-\left.z_{1}\right|^{2}} \\
& \leq 2 C_{1} 16 t^{2} \frac{1-|z|}{\left|1-\left\langle z, e_{1}\right\rangle\right|^{2}}=C_{2} t^{2} \frac{1-|z|}{|1-\langle z, \zeta\rangle|^{2}} .
\end{aligned}
$$

This completes the proof of (3.4) for $\zeta=e_{1}$. Now let $\zeta \in S$ be arbitrary, and let $\psi$ be the unitary transformation on $\mathbb{C}^{n}$ such that $\psi\left(e_{1}\right)=\zeta$. From the identity (2.1) we have $\left|\phi_{z}(\psi(w))\right|=\left|\phi_{\psi^{-1}(z)}(w)\right|$, and so the proof of (3.3) and (3.4) for arbitrary $\zeta$ reduces to the one for $\zeta=e_{1}$. 
Lemma 3.5. Let $t$ and $I_{t}$ be as in Lemma 3.4 and let $\zeta \in S$ and $h \in \mathcal{H}$ be such that

$$
r(\mu, \zeta, x) \leq h(x)
$$

for all $x>0$ and every non-negative regular Borel measure $\mu$ on $B$ satisfying the growth condition (2.2). Then there exists an absolute constant $C$ which does not depend on $t$ such that

$$
\inf _{r \in I_{t}}\left\{V_{\mu}(r \zeta)\right\} \leq C \frac{h(t)}{t} .
$$

Proof. From the definition of $G_{\zeta, t}$ and Fubini's theorem we have

$$
\begin{aligned}
\int_{B} G_{\zeta, t}(z) d \mu(z) & =\int_{I_{t}}\left(\int_{B} \log \frac{1}{\left|\phi_{z}(r \zeta)\right|} d \mu(z)\right) d r \\
& =\int_{I_{t}}\left(\int_{B} \log \frac{1}{\left|\phi_{r \zeta}(z)\right|} d \mu(z)\right) d r \\
& =\int_{I_{t}} V_{\mu}(r \zeta) d r \geq \int_{I_{t}}\left(\inf _{s \in I_{t}}\left\{V_{\mu}(s \zeta)\right\}\right) d r=t \inf _{s \in I_{t}} V_{\mu}(s \zeta) .
\end{aligned}
$$

Thus

$$
\inf _{r \in I_{t}}\left\{V_{\mu}(r \zeta)\right\} \leq \frac{1}{t} \int_{B} G_{\zeta, t}(z) d \mu(z)
$$

for every $t$ with $0<t<1 / 3$. Therefore from (3.2) and (3.3) it follows that

$$
\begin{aligned}
\inf _{r \in I_{t}}\left\{V_{\mu}(r \zeta)\right\} & \leq \frac{1}{t} \int_{B_{t}(\zeta)} G_{\zeta, t}(z) d \mu(z)+\frac{1}{t} \int_{B \backslash B_{t}(\zeta)} G_{\zeta, t}(z) d \mu(z) \\
& \leq \frac{C_{1}}{t} \int_{B_{t}(\zeta)}\left(1-|z|^{2}\right) d \mu(z)+C_{2} t \int_{B \backslash B_{t}(\zeta)} \frac{1-|z|^{2}}{|1-\langle z, \zeta\rangle|^{2}} d \mu(z) .
\end{aligned}
$$

If for $j=1,2, \ldots$ we set

$$
A_{j, t}(\zeta)=\left\{z \in B: 2^{j-1} t<|1-\langle z, \zeta\rangle| \leq 2^{j} t\right\},
$$

then from the last estimate and the definition $(2.3)$ of $r(\mu, \zeta, t)$ we have

$$
\begin{aligned}
\inf _{r \in I_{t}}\left\{V_{\mu}(r \zeta)\right\} & \leq \frac{C_{1}}{t} r(\mu, \zeta, t)+C_{2} t \sum_{j=1}^{\infty} \int_{A_{j, t}(\zeta)} \frac{1-|z|}{|1-\langle z, \zeta\rangle|^{2}} d \mu(z) \\
& \leq \frac{C_{1}}{t} r(\mu, \zeta, t)+C_{2} t \sum_{j=1}^{\infty} \int_{A_{j, t}(\zeta)} \frac{1-|z|}{t^{2} 2^{2 j-2}} d \mu(z) \\
& \leq \frac{C_{1}}{t} r(\mu, \zeta, t)+\frac{C_{2}}{t} \sum_{j=1}^{\infty} 2^{2-2 j} r\left(\mu, \zeta, 2^{j} t\right)=\frac{C}{t} h(t) .
\end{aligned}
$$


By hypothesis, $r(\mu, \zeta, x) \leq h(x)$ for every $x>0$, and therefore from the above it follows that

$$
\begin{aligned}
\inf _{r \in I_{t}}\left\{V_{\mu}(r \zeta)\right\} & \leq \frac{C_{1}}{t} h(t)+\frac{C_{2}}{t} \sum_{j=1}^{\infty} 2^{-2 j+2} h\left(2^{j} t\right) \\
& \leq \frac{C_{1}}{t} h(t)+\frac{C_{2}}{t} \sum_{j=1}^{\infty} 2^{-2 j+2} 2^{j} h(t)=\frac{C_{1}+C_{2}^{\prime}}{t} h(t) .
\end{aligned}
$$

Above we used the fact that $h(k x) \leq k h(x)$ for every $k \geq 1$ and every $x>0$. This follows easily from the hypothesis that the function $h(t) / t$ is non-increasing on the interval $(0, \infty)$.

Lemma 3.6. Let $\alpha>1 / 2$ be fixed. Then there exist two positive constants $C_{1}$ and $C_{2}$ such that

$$
C_{1} \liminf _{t \rightarrow 0+} \frac{r(\mu, \zeta, t)}{h(t)} \leq \liminf _{\substack{z \rightarrow \zeta \\ z \in D_{\alpha}(\zeta)}} \frac{|1-\langle z, \zeta\rangle|}{h(|1-\langle z, \zeta\rangle|)} V_{\mu}(z),
$$

and

$$
\liminf _{r \rightarrow 1-} \frac{1-r}{h(1-r)} V_{\mu}(r \zeta) \leq C_{2} \limsup _{t \rightarrow 0+} \frac{r(\mu, \zeta, t)}{h(t)}
$$

for all $\zeta \in S, h \in \mathcal{H}$, and all non-negative regular Borel measures $\mu$ satisfying the growth condition (2.2).

Proof. From the identity (2.1) and the inequality (3.2) we have

$$
\begin{aligned}
V_{\mu}(z) & =-\frac{1}{2} \int_{B} \log \left\{1-\frac{\left(1-|z|^{2}\right)\left(1-|w|^{2}\right)}{|1-\langle z, w\rangle|^{2}}\right\} d \mu(w) \\
& \geq \frac{1}{2} \int_{B} \frac{\left(1-|z|^{2}\right)\left(1-|w|^{2}\right)}{|1-\langle z, w\rangle|^{2}} d \mu(w) \\
& \geq \frac{1}{2} \int_{B_{t}(\zeta)} \frac{\left(1-|z|^{2}\right)\left(1-|w|^{2}\right)}{|1-\langle z, w\rangle|^{2}} d \mu(w),
\end{aligned}
$$

where $t=|1-\langle z, \zeta\rangle|$. Therefore, for all $z \in D_{\alpha}(\zeta)$ we have

$$
V_{\mu}(z) \geq \frac{|1-\langle z, \zeta\rangle|}{\alpha} \int_{B_{t}(\zeta)} \frac{(1-|w|)}{|1-\langle z, w\rangle|^{2}} d \mu(w) .
$$

Since $\sqrt{|1-\langle z, w\rangle|}$ satisfies the triangle inequality on $\bar{B} \times \bar{B}$ (Theorem K), for all $w \in B_{t}(\zeta)$ we have

$$
|1-\langle z, w\rangle| \leq 4|1-\langle z, \zeta\rangle| .
$$


Therefore, for all $z \in D_{\alpha}(\zeta)$, from (3.6) we have

$$
\begin{aligned}
V_{\mu}(z) & \geq \frac{|1-\langle z, \zeta\rangle|}{\alpha} \frac{1}{16|1-\langle z, \zeta\rangle|^{2}} \int_{B_{t}(\zeta)}(1-|w|) d \mu(w) \\
& \geq \frac{r(\mu, \zeta,|1-\langle z, \zeta\rangle|)}{32 \alpha|1-\langle z, \zeta\rangle|} .
\end{aligned}
$$

Hence, for all $z \in D_{\alpha}(\zeta)$,

$$
\frac{|1-\langle z, \zeta\rangle|}{h(|1-\langle z, \zeta\rangle|)} V_{\mu}(z) \geq C(\alpha) \frac{r(\mu, \zeta,|1-\langle z, \zeta\rangle|)}{h(|1-\langle z, \zeta\rangle|)},
$$

from which our estimate (a) easily follows.

To prove (b), we may assume that

$$
\limsup _{t \rightarrow 0+} \frac{r(\mu, \zeta, t)}{h(t)}=l, \quad 0 \leq l<\infty .
$$

Let $\varepsilon>0$ be given. Then there exists $t_{0}>0$ such that $r(\mu, \zeta, t)<(l+\varepsilon) h(t)$ for all $0<t \leq t_{0}$. Let $\mu_{1}$ be the restriction of $\mu$ to $B_{t_{0}}(\zeta)$, and let

$$
\begin{aligned}
& V_{1}(z)=\int_{B_{t_{0}}(\zeta)} \log \frac{1}{\left|\phi_{z}(w)\right|} d \mu(w)=\int_{B} \log \frac{1}{\left|\phi_{z}(w)\right|} d \mu_{1}(w), \\
& V_{2}(z)=\int_{B \backslash B_{t_{0}}(\zeta)} \log \frac{1}{\left|\phi_{z}(w)\right|} d \mu(w) .
\end{aligned}
$$

It is obvious that $V_{2}(r \zeta) \rightarrow 0$ as $r \rightarrow 1-$. Now consider $V_{1}$. Let $x>0$. If $x \leq t_{0}$, then

$$
r\left(\mu_{1}, \zeta, x\right)=r(\mu, \zeta, x)<(l+\varepsilon) h(x) .
$$

If $x>t_{0}$, then

$$
r\left(\mu_{1}, \zeta, x\right)=r\left(\mu, \zeta, t_{0}\right)<(l+\varepsilon) h\left(t_{0}\right) \leq(l+\varepsilon) h(x) .
$$

Therefore, for all $x>0$ we have

$$
r\left(\mu_{1}, \zeta, x\right) \leq(l+\varepsilon) h(x) .
$$

Applying now Lemma 3.5 to $\mu_{1}$ and $(l+\varepsilon) h(x)$ we obtain

$$
\begin{aligned}
\inf \left\{\frac{(1-r)}{h(1-r)} V_{\mu_{1}}(r \zeta): r \in I_{t}\right\} \leq \frac{3 t}{h(3 t)} \inf \left\{V_{\mu_{1}}(r \zeta): r \in I_{t}\right\} \\
\leq \frac{3 t}{h(3 t)} C_{1}(l+\varepsilon) \frac{h(t)}{t}=3 C_{1} \frac{h(t)}{h(3 t)}(l+\varepsilon) \leq 3 C_{1}(l+\varepsilon) .
\end{aligned}
$$

In the last step above we have used the fact that $h(t) \leq h(3 t)$.

Therefore

$$
\liminf _{r \rightarrow 1^{-}} \frac{1-r}{h(1-r)} V_{\mu_{1}}(r \zeta) \leq 3 C_{1}(l+\varepsilon),
$$


and since $\varepsilon>0$ was arbitrary it follows that

$$
\liminf _{r \rightarrow 1-} \frac{1-r}{h(1-r)} V_{\mu}(r \zeta) \leq 3 C_{1} l=3 C_{1} \limsup _{t \rightarrow 0+} \frac{r(\mu, \zeta, t)}{h(t)},
$$

as required.

Lemma 3.7. Let $h \in \mathcal{H}_{0}$. For a non-negative regular Borel measure $\mu$ on $B$ which satisfies the growth condition (2.2) let

$$
\overline{\mathcal{R}}_{0}(\mu, h)=\left\{\zeta \in S: \limsup _{t \rightarrow 0+} \frac{r(\mu, \zeta, t)}{h(t)}>0\right\} .
$$

Then

$$
H_{h}\left(\overline{\mathcal{R}}_{0}(\mu, h)\right)=0 .
$$

Proof. Since $H_{h}$ is subadditive it is enough to show that $H_{h}\left(\overline{\mathcal{R}}_{a}(\mu, h)\right)=0$ for all $a>0$, where for $a>0$,

$$
\overline{\mathcal{R}}_{a}(\mu, h)=\left\{\zeta \in S: \limsup _{t \rightarrow 0+} \frac{r(\mu, \zeta, t)}{h(t)}>a\right\} .
$$

Let $\varepsilon>0$ be given. From the growth condition (2.2) and the regularity of the measure $\mu$ it follows that there exists a compact set $K \subseteq B$ such that

$$
\int_{B \backslash K}\left(1-|w|^{2}\right) d \mu(w)<\varepsilon .
$$

Let $F$ be a compact subset of $\overline{\mathcal{R}}_{a}$, and let

$$
\mathcal{F}_{a}=\left\{Q(\zeta, 2 t): \frac{r(\mu, \zeta, t)}{h(t)}>a, \zeta \in F, 0<t<\varrho(K)\right\},
$$

where $\varrho(K)=\inf \{1-|z|: z \in K\}$. Notice that $\mathcal{F}_{a}$ is a covering of $F$ by Korányi balls. Since $F$ is a compact set, there exist points $\zeta_{1}, \ldots, \zeta_{m} \in S$ and positive numbers $t_{1}, \ldots, t_{m}$ such that

and

$$
F \subseteq \bigcup_{j=1}^{m} Q\left(\zeta_{j}, 2 t_{j}\right)
$$

$$
r\left(\mu, \zeta_{j}, t_{j}\right)>a h\left(t_{j}\right), \quad 0<t_{j}<\varrho(K) .
$$

By the covering Lemma 3.3, there exists a finite disjoint subcollection of $\mathcal{F}_{a}$, which for convenience we denote by $\left\{Q\left(\eta_{j}, 2 t_{j}\right): j=1, \ldots, k\right\}$, such that

$$
\begin{aligned}
& F \subseteq \bigcup_{j=1}^{k} Q\left(\eta_{j}, 18 t_{j}\right), \\
& r\left(\mu, \eta_{j}, t_{j}\right)>a h\left(t_{j}\right), \quad 0<t_{j}<\varrho(K), \quad j=1, \ldots, k .
\end{aligned}
$$


Now let $\nu$ be any positive measure on $S$ satisfying the condition (3.1) in Theorem 3.2:

$$
\nu(Q(\zeta, \delta)) \leq C h(\delta)
$$

for all $\zeta \in S$ and every $\delta>0$. Then from (3.9) we have

$$
\begin{aligned}
\nu(F) & \leq \sum_{j=1}^{k} \nu\left(Q\left(\eta_{j}, 18 t_{j}\right)\right) \leq C \sum_{j=1}^{k} h\left(18 t_{j}\right) \leq C \sum_{j=1}^{k} h\left(t_{j}\right) \\
& \leq \frac{C}{a} \sum_{j=1}^{k} r\left(\mu, \eta_{j}, t_{j}\right)=\frac{C}{a} \sum_{j=1}^{k} \int_{B_{t_{j}}\left(\eta_{j}\right)}\left(1-|w|^{2}\right) d \mu(w) .
\end{aligned}
$$

If $z \in B$ is such that $\left|1-\left\langle z, \eta_{j}\right\rangle\right| \leq t_{j}$ for some $j=1, \ldots, k$, then

$$
1-|z| \leq\left|1-\left\langle z, \eta_{j}\right\rangle\right| \leq t_{j}<\varrho(K) .
$$

Therefore

$$
\bigcup_{j=1}^{k} B_{t_{j}}\left(\eta_{j}\right) \subseteq \bigcup_{j=1}^{k}\left\{z \in B:\left|1-\left\langle z, \eta_{j}\right\rangle\right| \leq t_{j}\right\} \subseteq B \backslash K .
$$

From the trivial inequality $|1-\langle z, \zeta\rangle| \geq \frac{1}{2}|1-\langle z /|z|, \zeta\rangle|$ for $z \in B$ and $\zeta \in S$ we have

$$
\left\{z \in B:\left|1-\left\langle z, \eta_{j}\right\rangle\right| \leq t_{j}\right\} \subseteq\left\{z \in B:\left|1-\left\langle z /|z|, \eta_{j}\right\rangle\right| \leq 2 t_{j}\right\} .
$$

Since $\left\{Q\left(\eta_{j}, 2 t_{j}\right): j=1, \ldots, k\right\}$ is a pairwise disjoint family of Korányi balls, from the last inclusion it follows that the family $\left\{B_{t_{j}}\left(\eta_{j}\right): j=\right.$ $1, \ldots, k\}$ is also pairwise disjoint. Therefore, from (3.9) and (3.10) we have

$$
\begin{aligned}
\nu(F) & \leq C \sum_{j=1}^{k} \int_{B_{t_{j}}\left(\eta_{j}\right)}\left(1-|z|^{2}\right) d \mu(z)=C \int_{\bigcup_{j=1}^{k} B_{t_{j}}\left(\eta_{j}\right)}\left(1-|z|^{2}\right) d \mu(z) \\
& \leq C \int_{B \backslash K}\left(1-|z|^{2}\right) d \mu(z)<C \varepsilon .
\end{aligned}
$$

Since $\varepsilon>0$ was arbitrary we have $\nu(F)=0$ and hence Theorem 3.2 implies the result.

4. Main results. As immediate consequences of Lemmas 3.6 and 3.7 we have the following results:

THEOREM 4.1. If $\mu$ is a non-negative Borel regular measure on $B$ satisfying the usual growth condition

$$
\int_{B}\left(1-|w|^{2}\right) d \mu(w)<\infty
$$


then the set $\mathcal{L}_{0}(\mu, 1)$ is empty. If $h \in \mathcal{H}_{0}$, then

$$
H_{h}\left(\mathcal{L}_{0}(\mu, h)\right)=0 .
$$

Theorem 4.2. Let $h \in \mathcal{H}, 0<\alpha<1$ and let $\mu$ be a non-negative Borel regular measure on $B$ satisfying the growth condition

$$
\int_{B}\left(1-|w|^{2}\right) d \mu(w)<\infty .
$$

Then

$$
\underline{\mathcal{R}}(\mu, h) \subseteq \mathcal{L}_{\alpha}(\mu, h) \subseteq \mathcal{L}(\mu, h) \subseteq \overline{\mathcal{R}}(\mu, h) .
$$

For a non-negative regular Borel measure $\mu$ on $B$ satisfying the growth condition (2.2) let $F_{\mu}$ be the function on $B$ defined by

$$
F_{\mu}(z)=\left(1-|z|^{2}\right) \int_{B} \frac{1-|w|^{2}}{|1-\langle z, w\rangle|^{2}} d \mu(w) .
$$

If $d \mu(w)=f(w) d \lambda(w)$, where $f$ is a non-negative measurable function on $B$, then we denote $F_{\mu}$ by $F_{f}$.

The next two propositions will be needed for the proofs of our next results. They provide sufficient conditions for the existence of the $\mathcal{T}_{\tau}$-limit of $F_{\mu}$ and $F_{f}$ at a point $\zeta \in S$. Although their proofs are almost identical to those of Propositions 1 and 2 in [18], they are included here only for reasons of completeness.

Proposition 4.3. Let $\mu$ be a non-negative regular Borel measure on $B$ satisfying the growth condition (2.2). If $\zeta \in S$ is such that

$$
\int_{\mathcal{T}_{\tau, c}(\zeta)} \frac{\left(1-|w|^{2}\right)^{\alpha}}{|1-\langle w, \zeta\rangle|^{\tau \alpha}} d \mu(w)<\infty
$$

and

$$
\int_{B \backslash \mathcal{T}_{\tau, c}(\zeta)} \frac{\left(1-|w|^{2}\right)^{\gamma}}{\mid 1-\langle w, \zeta\rangle^{\tau \gamma}} d \mu(w)<\infty
$$

for some $c>0, \alpha>0, \gamma<1$, and $\tau \geq 1$, then

$$
\lim _{\substack{z \rightarrow \zeta \\ z \in \mathcal{T}_{\tau, c}(\zeta)}} F_{\mu}(z)=0 .
$$

Proof. For $0<R<1$, let $A_{R}=\{z: R<|z|<1\}$. Then

$$
\begin{aligned}
& F_{\mu}(z) \leq C(R)\left(1-|z|^{2}\right)+\left(1-|z|^{2}\right) \int_{A_{R}} \frac{1-|w|^{2}}{|1-\langle z, w\rangle|^{2}} d \mu(w) \\
& =C(R)\left(1-|z|^{2}\right)+\left(1-|z|^{2}\right) \int_{A_{R} \cap \mathcal{T}_{\tau, c}(\zeta)} \frac{\left(1-|w|^{2}\right)^{\alpha}\left(1-|w|^{2}\right)^{1-\alpha}}{|1-\langle z, w\rangle|^{2-\alpha}|1-\langle z, w\rangle|^{\alpha}} d \mu(w)
\end{aligned}
$$




$$
\begin{aligned}
& \quad+\left(1-|z|^{2}\right) \int_{A_{R} \backslash \mathcal{T}_{\tau, c}(\zeta)} \frac{\left(1-|w|^{2}\right)^{\gamma}\left(1-|w|^{2}\right)^{1-\gamma}}{|1-\langle z, w\rangle|^{2-\gamma}|1-\langle z, w\rangle|^{\gamma}} d \mu(w) \\
& \leq C(R)\left(1-|z|^{2}\right)+C \int_{A_{R} \cap \mathcal{T}_{\tau, c}(\zeta)} \frac{\left(1-|w|^{2}\right)^{\alpha}}{|1-\langle z, w\rangle|^{\alpha}} d \mu(w) \\
& +C \int_{A_{R} \backslash \mathcal{T}_{\tau, c}(\zeta)} \frac{\left(1-|w|^{2}\right)^{\gamma}}{|1-\langle z, w\rangle|^{\gamma}} d \mu(w) .
\end{aligned}
$$

Now let $z \in \mathcal{T}_{\tau, c}(\zeta)$ and let $w \in B$. Then

$$
|1-\langle z, \zeta\rangle|^{\tau} \leq c\left(1-|z|^{2}\right) \leq(2 c)|1-\langle z, w\rangle| .
$$

Using this and the fact that the function $\sqrt{|1-\langle z, w\rangle|}$ satisfies the triangle inequality on $\bar{B} \times \bar{B}$ (Theorem $\mathrm{K}$ ) we see that for all $z \in \mathcal{T}_{\tau, c}(\zeta)$ and all $w \in B$,

$$
\begin{aligned}
|1-\langle w, \zeta\rangle| & \leq 2(|1-\langle w, z\rangle|+|1-\langle z, \zeta\rangle|) \\
& \leq 2|1-\langle w, z\rangle|+2(2 c)^{1 / \tau}|1-\langle w, z\rangle|^{1 / \tau} \\
& \leq C(c, \tau)|1-\langle w, z\rangle|^{1 / \tau} .
\end{aligned}
$$

Thus

$$
|1-\langle w, \zeta\rangle|^{\tau} \leq C(c, \tau)|1-\langle w, z\rangle|
$$

for all $z \in \mathcal{T}_{\tau, c}(\zeta)$ and $w \in B$, and therefore by (4.4),

$$
\begin{aligned}
F_{\mu}(z) \leq & C(R)\left(1-|z|^{2}\right)+C(c, \tau) \int_{A_{R} \cap \mathcal{T}_{\tau, c}(\zeta)} \frac{\left(1-|w|^{2}\right)^{\alpha}}{|1-\langle w, \zeta\rangle|^{\alpha \tau}} d \mu(w) \\
& +C(c, \tau) \int_{A_{R} \backslash \mathcal{T}_{\tau, c}(\zeta)} \frac{\left(1-|w|^{2}\right)^{\gamma}}{|1-\langle w, \zeta\rangle|^{\gamma \tau}} d \mu(w)
\end{aligned}
$$

for all $z \in \mathcal{T}_{\tau, c}(\zeta)$.

As a consequence of hypotheses (4.2) and (4.3) we have $\lim _{R \rightarrow 1} \int_{A_{R} \cap \mathcal{T}_{\tau, c}(\zeta)} \frac{\left(1-|w|^{2}\right)^{\alpha}}{\mid 1-\left\langle w,\left.\zeta\right|^{\alpha \tau}\right.} d \mu(w)=\lim _{R \rightarrow 1} \int_{A_{R} \backslash \mathcal{T}_{\tau, c}(\zeta)} \frac{\left(1-|w|^{2}\right)^{\gamma}}{|1-\langle w, \zeta\rangle|^{\gamma \tau}} d \mu(w)=0$.

Therefore, for any $\varepsilon>0$, we can find $R$ with $0<R<1$ such that

$$
\begin{aligned}
C(c, \tau) \int_{A_{R} \cap \mathcal{T}_{\tau, c}(\zeta)} & \frac{\left(1-|w|^{2}\right)^{\alpha}}{|1-\langle w, \zeta\rangle|^{\alpha \tau}} d \mu(w) \\
+C(c, \tau) \int_{A_{R} \backslash \mathcal{T}_{\tau, c}(\zeta)} & \frac{\left(1-|w|^{2}\right)^{\gamma}}{|1-\langle w, \zeta\rangle|^{\gamma \tau}} d \mu(w)<\varepsilon .
\end{aligned}
$$


Hence, for this $R$, from (4.5) we have

$$
\lim _{\substack{z \rightarrow \zeta \\ z \in \mathcal{T}_{\tau, c}(\zeta)}} F_{\mu}(z)<\varepsilon
$$

which proves the result.

Proposition 4.4. Let $f$ be a non-negative measurable function on $B$. If $\zeta \in S$ and $c>0, \tau \geq 1$ are such that

$$
\int_{\mathcal{T}_{\tau, c}(\zeta)} \frac{\left(1-|w|^{2}\right)^{\alpha}}{|1-\langle w, \zeta\rangle|^{\tau \alpha}} f^{p}(w) d \lambda(w)<\infty
$$

and

$$
\int_{B \backslash \mathcal{T}_{\tau, c}(\zeta)} \frac{\left(1-|w|^{2}\right)^{\gamma}}{|1-\langle w, \zeta\rangle|^{\tau \gamma}} f^{p}(w) d \lambda(w)<\infty
$$

for some $p$ with $1<p<n /(n-1)$, and some $\alpha$ and $\gamma$ with $0<\alpha<n+p-n p$ and $0<\gamma<n+p-n p$, then

$$
\lim _{\substack{z \rightarrow \zeta \\ z \in \mathcal{T}_{\tau, c}(\zeta)}} F_{f}(z)=0
$$

Proof. As in Proposition 4.2, for $z \in B$ and $0<R<1$ we have

$$
\begin{aligned}
F_{f}(z) \leq & C(R)\left(1-|z|^{2}\right)+\left(1-|z|^{2}\right) \int_{A_{R}} \frac{1-|w|^{2}}{|1-\langle z, w\rangle|^{2}} f(w) d \lambda(w) \\
= & C(R)\left(1-|z|^{2}\right)+\left(1-|z|^{2}\right) \int_{A_{R} \cap \mathcal{T}_{\tau, c}(\zeta)} \frac{1-|w|^{2}}{|1-\langle z, w\rangle|^{2}} f(w) d \lambda(w) \\
& +\left(1-|z|^{2}\right) \int_{A_{R} \backslash \mathcal{T}_{\tau, c}(\zeta)} \frac{1-|w|^{2}}{|1-\langle z, w\rangle|^{2}} f(w) d \lambda(w) .
\end{aligned}
$$

If $q=p /(p-1)$ is the conjugate exponent of $p$, then by Hölder's inequality,

$$
\begin{aligned}
F_{f}(z) \leq & C(R)\left(1-|z|^{2}\right)+\left(1-|z|^{2}\right)\left\{\int_{A_{R}} \frac{\left(1-|w|^{2}\right)^{(1-\alpha / p) q}}{|1-\langle z, w\rangle|^{(2-\alpha / p) q}} d \lambda(w)\right\}^{1 / q} \\
& \times\left\{\int_{A_{R} \cap \mathcal{T}_{\tau, c}(\zeta)} \frac{\left(1-|w|^{2}\right)^{\alpha}}{|1-\langle z, w\rangle|^{\alpha}} f^{p}(w) d \lambda(w)\right\}^{1 / p} \\
& +\left(1-|z|^{2}\right)\left\{\int_{A_{R}} \frac{\left(1-|w|^{2}\right)^{(1-\gamma / p) q}}{|1-\langle z, w\rangle|^{(2-\gamma / p) q}} d \lambda(w)\right\}^{1 / q} \\
& \times\left\{\int_{A_{R} \backslash \mathcal{T}_{\tau, c}(\zeta)} \frac{\left(1-|w|^{2}\right)^{\gamma}}{|1-\langle z, w\rangle|^{\gamma}} f^{p}(w) d \lambda(w)\right\}^{1 / p} .
\end{aligned}
$$


If we apply to this estimate the asymptotic estimate from Proposition 3.1 ,

$$
\int_{A_{R}} \frac{\left(1-|w|^{2}\right)^{(1-k / p) q}}{|1-\langle z, w\rangle|^{(2-k / p) q}} d \lambda(w) \leq C\left(1-|z|^{2}\right)^{-q}, \quad k=\alpha \quad \text { or } \quad k=\gamma
$$

provided $(1-k / p) q>n$, i.e., $k<n+p-n p$, we have

$$
\begin{aligned}
F_{f}(z) \leq & C(R)\left(1-|z|^{2}\right)+C\left\{\int_{A_{R} \cap \mathcal{T}_{\tau, c}(\zeta)} \frac{\left(1-|w|^{2}\right)^{\alpha}}{|1-\langle z, w\rangle|^{\alpha}} f^{p}(w) d \lambda(w)\right\}^{1 / p} \\
& +C\left\{\int_{A_{R} \backslash \mathcal{T}_{\tau, c}(\zeta)} \frac{\left(1-|w|^{2}\right)^{\gamma}}{|1-\langle z, w\rangle|^{\gamma}} f^{p}(w) d \lambda(w)\right\}^{1 / p} .
\end{aligned}
$$

As in Proposition 4.3 we have

$$
|1-\langle w, z\rangle| \geq C(c, \tau)|1-\langle w, \zeta\rangle|^{\tau}
$$

for all $z \in \mathcal{T}_{\tau, c}(\zeta)$ and all $w \in B$. Therefore, for $z \in \mathcal{T}_{\tau, c}(\zeta)$ from (4.8) it follows that

$$
\begin{aligned}
F_{f}(z) \leq & C(R)\left(1-|z|^{2}\right) \\
& +C(c, \tau)\left\{\int_{A_{R} \cap \mathcal{T}_{\tau, c}(\zeta)} \frac{\left(1-|w|^{2}\right)^{\alpha}}{|1-\langle w, \zeta\rangle|^{\tau \alpha}} f^{p}(w) d \lambda(w)\right\}^{1 / p} \\
& +C(c, \tau)\left\{\int_{A_{R} \backslash \mathcal{T}_{\tau, c}(\zeta)} \frac{\left(1-|w|^{2}\right)^{\gamma}}{|1-\langle w, \zeta\rangle|^{\tau \gamma}} f^{p}(w) d \lambda(w)\right\}^{1 / p},
\end{aligned}
$$

and so hypotheses (4.6) and (4.7) give the proof as in Proposition 4.3.

The proof of the following proposition is omitted since it closely parallels the proof of a similar result, Proposition 3, given in [18, pp. 148-151].

Proposition 4.5. Let $\mu$ be a non-negative regular Borel measure on $B$ satisfying

$$
\int_{B}\left(1-|w|^{2}\right)^{\beta} d \mu(w)<\infty
$$

for some $\beta$ with $0<\beta<1$, and let $1 \leq \tau \leq n / \beta$. Then

(a) for any $\gamma$ with $\beta<\gamma \leq 1$,

$$
H_{\beta \tau}\left(\left\{\zeta \in S: \int_{B \backslash \mathcal{I}_{\tau, c}(\zeta)} \frac{\left(1-|w|^{2}\right)^{\gamma}}{|1-\langle w, \zeta\rangle|^{\tau \gamma}} d \mu(w)=\infty\right\}\right)=0
$$

(b) for any $\alpha$ with $0<\alpha<\beta$,

$$
H_{\beta \tau}\left(\left\{\zeta \in S: \int_{\mathcal{T}_{\tau, c}(\zeta)} \frac{\left(1-|w|^{2}\right)^{\alpha}}{|1-\langle w, \zeta\rangle|^{\tau \alpha}} d \mu(w)=\infty\right\}\right)=0 .
$$


From the above propositions we have the following

COROLlary 4.6. If $\mu$ is a non-negative regular Borel measure on $B$ satisfying

$$
\int_{B}\left(1-|w|^{2}\right)^{\beta} d \mu(w)<\infty
$$

for some $\beta$ with $0<\beta<1$, then for each $\tau$ with $1 \leq \tau \leq n / \beta$ there exists a set $E_{\tau} \subseteq S$ with $H_{\beta \tau}\left(E_{\tau}\right)=0$ such that $F_{\mu}$ has $\mathcal{T}_{\tau}$-limit 0 at all points $\zeta \in S \backslash E_{\tau}$.

Proof. Fix $\alpha$ and $\gamma$ satisfying $0<\alpha<\beta<\gamma \leq 1$. By Proposition 4.5, there exists a set $E_{\tau} \subseteq S$ with $H_{\beta \tau}\left(E_{\tau}\right)=0$ such that the integrals in (a) and (b) of Proposition 4.5 are finite for all $\zeta \in S \backslash E_{\tau}$. For those $\zeta$, now apply Proposition 4.3.

COROLlaRY 4.7. If $\mu$ is a non-negative regular Borel measure on B satisfying the condition

$$
\int_{B}\left(1-|w|^{2}\right)^{\beta} d \mu(w)<\infty,
$$

for some $\beta$ with $0<\beta<1$, then for each $\tau$ with $1 \leq \tau \leq n / \beta$,

$$
H_{\beta \tau}\left(\left\{\zeta \in S: \mu\left(\mathcal{T}_{\tau, c}(\zeta)\right)=\infty\right\}\right)=0 .
$$

Proof. Since $|1-\langle w, \zeta\rangle|^{\tau}<c\left(1-|w|^{2}\right)$ for all $w \in \mathcal{T}_{\tau, c}(\zeta)$,

$$
\int_{\mathcal{T}_{\tau, c}(\zeta)} \frac{\left(1-|w|^{2}\right)^{\beta}}{|1-\langle w, \zeta\rangle|^{\tau \beta}} d \mu(w) \geq C \mu\left(\mathcal{T}_{\tau, c}(\zeta)\right) .
$$

The result is now an immediate consequence of Proposition 4.4(b).

Now we are ready for our next main theorems.

TheOREM 4.8. Let $f$ be a non-negative measurable function on $B$ satisfying

$$
\int_{B}\left(1-|z|^{2}\right)^{\alpha} f^{p}(z) d \lambda(z)<\infty
$$

for some $p$ with $1<p<n /(n-1)$ and some $\alpha$ with $0<\alpha<n+p-n p$. Then for each $\tau$ with $1 \leq \tau \leq n / \alpha$ there exists a set $E_{\tau} \subseteq S$ with $H_{\alpha \tau}\left(E_{\tau}\right)=0$ such that $V_{f}$ has $\mathcal{T}_{\tau}$-limit 0 at all points $\zeta \in S \backslash E_{\tau}$.

Proof. For a function $f$ which satisfies (4.9) let

$$
\begin{aligned}
& V_{1}(z)=\int_{E(z)} \log \frac{1}{\left|\phi_{z}(w)\right|} f(w) d \lambda(w), \\
& V_{2}(z)=\int_{B \backslash E(z)} \log \frac{1}{\left|\phi_{z}(w)\right|} f(w) d \lambda(w) .
\end{aligned}
$$

Recall that $E(z)=\left\{w \in B:\left|\phi_{z}(w)\right|<1 / 2\right\}$. 
From the inequality (3.2) it follows that

$$
\log \frac{1}{|z|} \leq C\left(1-|z|^{2}\right) \quad \text { for all } z \in B \text { with }|z| \geq 1 / 2 .
$$

Therefore from the identity (2.1) we have

$$
\log \frac{1}{\left|\phi_{z}(w)\right|} \leq C \frac{\left(1-|z|^{2}\right)\left(1-|w|^{2}\right)}{|1-\langle w, z\rangle|^{2}}
$$

for all $w \in B \backslash E(z)$. Thus,

$$
V_{2}(z) \leq C\left(1-|z|^{2}\right) \int_{B} \frac{1-|w|^{2}}{|1-\langle w, z\rangle|^{2}} f(w) d \lambda(w)=C F_{f}(z)
$$

for all $z \in B$.

If we take $d \mu(w)=f^{p}(w) d \lambda(w)$, then from Propositions 4.4 and 4.5 it follows that there exists a set $E_{2} \subseteq S$ such that $H_{\alpha \tau}\left(E_{2}\right)=0$ and $F_{f}$ has $\mathcal{T}_{\tau}$-limit 0 at all points $\zeta \in S \backslash E_{2}$. Therefore there exists a set $E_{2} \subseteq S$ such that $H_{\alpha \tau}\left(E_{2}\right)=0$ and $V_{2}$ has $\mathcal{T}_{\tau}$-limit 0 at all points $\zeta \in S \backslash E_{2}$.

Now consider the function $V_{1}$. Taking $d \mu(w)=f^{p}(w) d \lambda(w)$ in Corollary 4.5, it follows that there exists a set $E_{1} \subseteq S$ with $H_{\alpha \tau}\left(E_{1}\right)=0$ such that

$$
\int_{\mathcal{I}_{\tau, c^{\prime}}(\zeta)} f^{p}(w) d \lambda(w)<\infty
$$

for all $\zeta \in S \backslash E_{1}$ and any $c^{\prime}>0$. Suppose that $c>0$ and $z \in \mathcal{T}_{\tau, c}(\zeta)$. Then by [19, Lemma 8.17] we have

$$
E(z) \subseteq \mathcal{T}_{\tau, c^{\prime}}(\zeta) \cap A_{r} \quad \text { for every } c^{\prime}>c 2^{\tau+1} \text { and } r \geq 2|z|^{2}-1 .
$$

Let $q$ be the conjugate exponent of $p$. From the above inclusion, the $\mathcal{M}$-invariance of $\lambda$ and Hölder's inequality we have

$$
\begin{aligned}
V_{1}(z) & \leq\left\{\int_{E(z)}\left(\log \frac{1}{\left|\phi_{z}(w)\right|}\right)^{q} d \lambda(w)\right\}^{1 / q}\left\{\int_{E(z)} f^{p}(w) d \lambda(w)\right\}^{1 / p} \\
& =\left\{\int_{\{w:|w|<1 / 2\}}\left(\log \frac{1}{\left|\phi_{z}(w)\right|}\right)^{q} d \lambda(w)\right\}^{1 / q}\left\{\int_{E(z)} f^{p}(w) d \lambda(w)\right\}^{1 / p} \\
& \leq C\left\{\int_{\mathcal{T}_{\tau, c^{\prime}}(\zeta) \cap A_{r}} f^{p}(w) d \lambda(w)\right\}^{1 / p},
\end{aligned}
$$

where $C$ is a constant which is independent of $z$ tending to the boundary of $B$.

Therefore, for all $z \in B$ we have

$$
V_{1}(z) \leq C\left\{\int_{\mathcal{T}_{\tau, c^{\prime}}(\zeta) \cap A_{r}} f^{p}(w) d \lambda(w)\right\}^{1 / p} .
$$


Now, as a consequence of (4.10) the above integral goes to 0 as $r \rightarrow 1$. Thus $V_{1}$ has $\mathcal{T}_{\tau}$-limit 0 at all points $\zeta \in S \backslash E_{1}$. If we take $E_{\tau}=E_{1} \cup E_{2}$, then $H_{\beta \tau}\left(E_{\tau}\right)=0$ and $V_{f}$ has $\mathcal{I}_{\tau}$-limit 0 at all points $\zeta \in S \backslash E_{\tau}$.

TheOREM 4.9. Let $\left\{z_{j}: j=1,2, \ldots\right\}$ be a sequence in $B$ satisfying

$$
\sum_{j=1}^{\infty}\left(1-\left|z_{j}\right|^{2}\right)^{\alpha}<\infty
$$

for some $0<\alpha<1$, and let $\mu$ be the measure given by $\mu=\sum \delta_{z_{j}}$, where $\delta_{z_{j}}$ is the unit point-mass measure at $z_{j}$. Then for each $\tau$ with $1 \leq \tau \leq n / \alpha$, there exists a set $E_{\tau} \subseteq S$ with $H_{\alpha \tau}\left(E_{\tau}\right)=0$ such that $V_{\mu}$ has $\mathcal{T}_{\tau}$-limit 0 at all points $\zeta \in S \backslash E_{\tau}$.

Proof. As in Theorem 4.8, for the measure $\mu$, let

$$
V_{1}(z)=\int_{E(z)} \log \frac{1}{\left|\phi_{z}(w)\right|} d \mu(w), \quad V_{2}(z)=\int_{B \backslash E(z)} \log \frac{1}{\left|\phi_{z}(w)\right|} d \mu(w) .
$$

As in Theorem 4.8, for the function $F_{\mu}$ defined by (4.1), we have

$$
V_{2}(z) \leq C F_{\mu}(z)
$$

for all $z \in B$, where $C$ is a constant independent on $z$.

By Corollary 4.5 there exists a set $E_{1} \subseteq S$ such that $H_{\beta \tau}\left(E_{1}\right)=0$ and $F_{\mu}$ has $\mathcal{T}_{\tau}$-limit 0 at all points $\zeta \in S \backslash E_{1}$. Therefore $V_{2}$ has $\mathcal{T}_{\tau}$-limit 0 at all points $\zeta \in S \backslash E_{1}$. By Corollary 4.6 there exists a set $E_{2} \subseteq S$ such that $H_{\alpha \tau}\left(E_{2}\right)=0$ and

$$
\mu\left(\mathcal{T}_{\tau, c}(\zeta)\right)<\infty \quad \text { for all } \zeta \in S \backslash E_{2} .
$$

Now, it is obvious that $\mu\left(\mathcal{T}_{\tau, c}(\zeta)\right)<\infty$ if and only if $\mathcal{T}_{\tau, c}(\zeta)$ contains only a finite number of terms of the sequence $\left\{z_{k}\right\}$. But for those $\zeta$ for which $\mu\left(\mathcal{T}_{\tau, c}(\zeta)\right)<\infty$ we obviously have

$$
\lim _{\substack{z \rightarrow \zeta \\ z \in \mathcal{T}_{\tau, c}(\zeta)}} V_{1}(z)=0
$$

which means that $V_{1}$ has $\mathcal{T}_{\tau}$-limit 0 at all points $\zeta \in S \backslash E_{2}$. Now take $E_{\tau}=E_{1} \cup E_{2}$.

Acknowledgements. I would like to thank Professor Manfred Stoll for his guidance and for many fruitful discussions during the preparation of the paper. I would also like to express my gratitude to the referee of the paper for many valuable comments and suggestions. The referee pointed out several errors and oversights (the proof of Lemma 3.7, in particular) in the earlier version of the paper. The errors and oversights have been corrected and all referee's comments and suggestions have been incorporated in the new version of the paper. 
K. Adzievski

\section{References}

[1] K. Adzievski, Boundary behavior of pluri-Green potentials on the unit ball B in $\mathbb{C}^{n}$, Complex Variables 32 (1995), 32-43.

[2] P. Ahern, Exceptional sets for holomorphic Sobolev functions, Michigan Math. J. 35 (1988), 29-41.

[3] P. Ahern and C. Cascante, Exceptional sets for Poisson integrals of potentials on the unit sphere in $\mathbb{C}^{n}, p \leq 1$, Pacific J. Math. 153 (1992), 11-13.

[4] P. Ahern and W. Cohn, Exceptional sets for Hardy Sobolev functions, $p>1$, Indiana Univ. Math. J. 38 (1989), 417-453.

[5] -, 一, Weighted maximal functions and derivatives of invariant Poisson integrals of potentials, Pacific J. Math. 163 (1994), 1-16.

[6] P. Ahern and A. Nagel, Strong $L^{p}$-estimates for maximal functions with respect to singular measures; with applications to exceptional sets, Duke Math. J. 53 (1986), 359-393.

[7] R. D. Berman and W. S Cohn, Tangential limits of Blaschke products and functions of bounded mean oscillation, Illinois J. Math. 31 (1987), 218-239.

[8] G. T. Cargo, Angular and tangential limits of Blaschke products and their successive derivatives, Canad. J. Math. 14 (1962), 334-348.

[9] C. Cascante and J. Ortega, A characterization of tangential exceptional sets for $H_{\alpha}^{p}$, $\alpha=n$, Proc. R. Soc. Edinb. Sect. A 126 (1996), 625-642.

[10] P. Cifuentes, J. R. Dorronsoro and J. Sueiro, Boundary tangential convergence on spaces of homogeneous type, Trans. Amer. Math. Soc. 332 (1992), 331-350.

[11] W. Cohn, Non-isotropic Hausdorff measure and exceptional sets for holomorphic Sobolev functions, Illinois J. Math. 33 (1989), 673-690.

[12] Ph. Jamming and M. M. Roginskaya, Boundary behavior of $\mathcal{M}$-harmonic functions and non-isotropic Hausdorff measure, Monatsh. Math. 134 (2002), 217-226.

[13] M. M. Roginskaya, Asymptotical properties of harmonic and $\mathcal{M}$-harmonic functions near the boundary of the unit sphere, Zap. Nauchn. Sem. POMI 270 (2000), 309-316 (in Russian).

[14] W. Rudin, Function Theory in the Unit Ball of $\mathbb{C}^{n}$, Springer, New York, 1980.

[15] Å. Samuelsson, On radial zeros of Blaschke products, Ark. Mat. 7 (1969), 477-494.

[16] T. Sjödin, A note on capacity and Hausdorff measure on homogeneous spaces, Potential Anal. 6 (1977), 87-97.

[17] M. Stoll, Boundary limits and non-integrability of $\mathcal{M}$-subharmonic functions on the unit ball of $\mathbb{C}^{n}(n \geq 1)$, Trans. Amer. Math. Soc. 349 (1997), 3773-3785.

[18] - Non-isotropic Hausdorff capacity of exceptional sets of invariant potentials, Potential Anal. 4 (1995), 141-155.

[19] -, Potential Theory in the Unit Ball of $\mathbb{C}^{n}$, London Math. Soc. Lecture Note Ser. 199, Cambridge Univ. Press, 1994.

[20] J. Sueiro, Tangential boundary limits and exceptional sets for holomorphic functions in Dirichlet-type spaces, Math. Ann. 286 (1990), 661-678.

Department of Mathematics and Computer Science

South Carolina State University

Orangeburg, SC 29117, U.S.A.

E-mail: kadzievski@scsu.edu 